\title{
The spectral energy distributions of the entire Herschel Reference Survey
}

\author{
Laure Ciesla ${ }^{1}$ and the Herschel-SPIRE Local Galaxies Guaranteed \\ Time Programs \\ ${ }^{1}$ Laboratoire d'Astrophysique de Marseille, UMR6110 CNRS, \\ 38 rue F. Joliot-Curie, F-13388 Marseille France \\ email: laure.ciesla@oamp.fr
}

\begin{abstract}
We present the spectral energy distributions (SED) of the 323 galaxies of the Herschel Reference Survey. In order to provide templates for nearby galaxies calibrated on physical parameters, we computed mean SEDs per bin of morphological types and stellar masses. They will be very useful to study more distant galaxies and their evolution with redshift. This preliminary work aims to study how the most commonly used libraries (Chary \& Elbaz 2001, Dale \& Helou 2002 and Draine \& Li 2007) reproduce the far-infrared emission of galaxies. First results show that they reproduce well the far-infrared part of mean SEDs. For single galaxies the Draine \& Li (2007) models seem to reproduce very well the far-infrared emission, as does the Dale \& Helou (2002).
\end{abstract}

Keywords. infrared: galaxies, galaxies: statistics, galaxies: elliptical and lenticular, galaxies: spiral

\section{Introduction}

The spectral energy distributions (SEDs) of galaxies are our primary source of information about their physical properties. All the different physical processes occurring in galaxies leave their imprint on the global and detailed shape of the SED, each dominating at different wavelengths. Dust is produced by the aggregation of metals injected into the interstellar medium by massive stars (through stellar winds). It absorbs the stellar light and re-emits it in the infrared, from $5 \mu \mathrm{m}$ to $1 \mathrm{~mm}$. IRAS (Neugebauer et al. 1984), ISO (Kessler et al. 1996) and Spitzer (Werner et al. 2004) provided us with data up to $200 \mu \mathrm{m}$ allowing us to study the emission of the warm dust. The Herschel space observatory (Pilbratt et al. 2010), launched in May 2009, opened a new window on the far infrared (55 to $672 \mu \mathrm{m})$. This new domain of the SED is fundamental to study the emission from cold dust, which dominates by mass. To study the properties of the cold dust, we built up a statistically complete, K-band-selected and volume-limited sample: the guaranteed time key project Herschel Reference Survey (HRS; PI: Eales. S.; Boselli et al. 2010a) which offers an unique view from $250 \mu \mathrm{m}$ to $500 \mu \mathrm{m}$ of nearby galaxies.

\section{SEDs of the HRS galaxies: data and mean SEDs}

Since HRS galaxies are well known nearby galaxies, HRS benefits from a large amount of ancillary data collected at other wavelengths (ultraviolet: GALEX; optical: SDSS; near-infrared: 2MASS, Spitzer/IRAC; radio) indispensable to extend the study of this proceeding to the whole spectrum in a future work. We focus only on the infrared part of the SED from 24 to $500 \mu \mathrm{m}$. We use IRAS 60 and $100 \mu \mathrm{m}$ data collected from different sources (Boselli et al. 2010a) and from NED. Spitzer/MIPS data come from Bendo et al. 

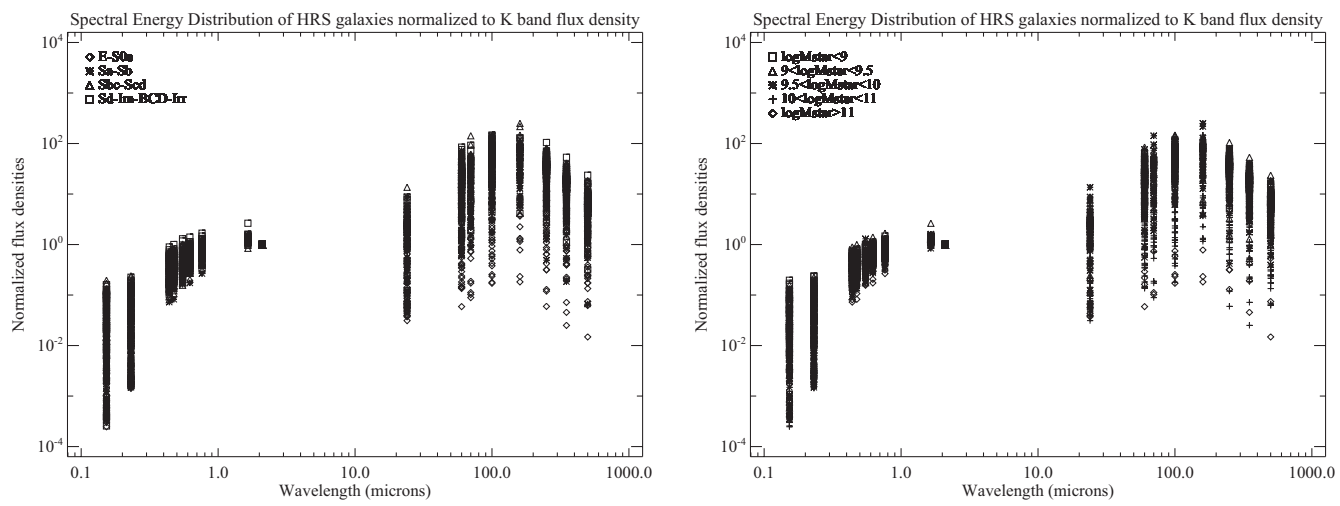

Figure 1. Spectral Energy Distributions of all the HRS galaxies normalized to the K band flux densities. Left panel: Galaxies are coded according to morphological type. Right panel: Galaxies are coded according to stellar mass.
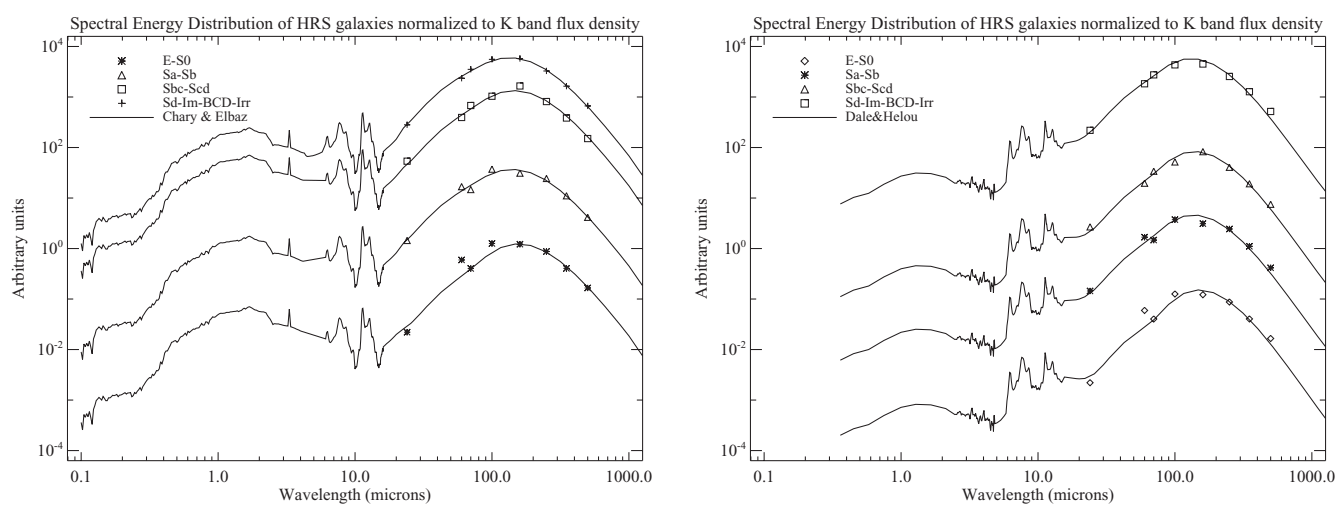

Figure 2. SED fitting of the galaxies binned by morphological type using Chary \& Elbaz (2001) (left panel) and Dale \& Helou (2002) (right panel).

(2011), who collected and performed the photometry of 168 HRS galaxies at 24, 70 and $160 \mu \mathrm{m}$. We have Herschel/SPIRE data for all 323 galaxies at 250, 350 and $500 \mu \mathrm{m}$. The photometry is fully described in Ciesla et al. (2012).

Because these SEDs come from a complete statistically sample, they are ideal to construct templates as a function of different parameters (morphological type, stellar mass, dust mass, specific star formation rate (sSFR), interstellar radiation field intensity, environment). In this very preliminary work, we focus on the morphological type and the stellar mass. Figure 1 shows the SEDs of all the HRS galaxies normalized to the K-band flux densities, per bins of morphological type (left panel) and stellar mass (right panel). For a first approach, we treat upper limits as real measurements of flux densities, and calculate the mean SED for each bin of morphological type and stellar mass.

\section{Comparison with literature}

We compare our observed SEDs with the popular infrared templates of Dale \& Helou (2002) and Chary \& Elbaz (2001). As shown on Figure 2, both libraries reproduce well the mean SEDs. Indeed, the particularities that single galaxies have is smoothed by computing the mean flux density in the bins. Elbaz et al. (2011) proposed two templates: one for starburst and one for main sequence (normal) galaxies. We find that the main 


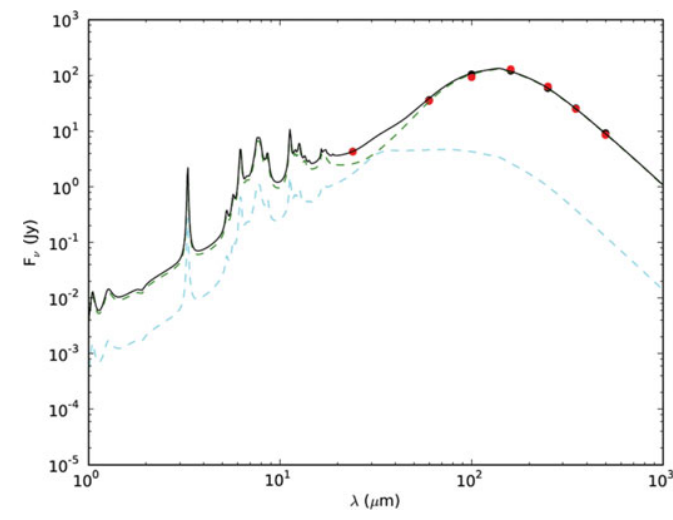

Figure 3. SED fitting of M99 (HRS102) using Draine \& Li (2007) models. The parameters of the model are the dust composition (LMC is used in this example), the minimum intensity of the radiation field, the fraction of the total dust mass that is heated by the distribution of starlight intensities, and the dust mass.

sequence template reproduces well the far-infrared part of the mean SED of late-type galaxies, unlike the starburst template. This is to be expected as the HRS sample doesn't contain strong starburst galaxies. However, fitting the two libraries on every single SED, we find that the Dale \& Helou (2002) library reproduces better the far-infrared emission of late-type galaxies than the Chary \& Elbaz (2001) one. We are now starting to fit the Draine \& Li (2007) models to the single SEDs (example with M99 in Figure 3). First preliminary results show that Draine \& Li (2007) models are the most appropriate models to reproduce the far-infrared emission of our galaxies.

\section{Conclusion}

HRS is a complete sample, small enough to study the galaxies one by one and large enough to permit statistical studies. First results on SED fitting show that the Dale \& Helou (2002) library seems to reproduce well the FIR SEDs of late types. While the Chary \& Elbaz (2001) library also seems to reproduce well the mean SEDs of late types, a further investigation has to be made with single galaxies. The Elbaz et al. (2011) main sequence template reproduces well the mean SEDs of late types. First preliminary results show that Draine \& Li (2007) seems to better reproduce HRS SEDs of late types.

\section{References}

Boselli, A., Eales, S., Cortese, L. et al. 2010a, PASP, 122, 261

Bendo G., Galliano F. \& Madden, S. 2011, MNRAS (in preparation)

Chary, R. \& Elbaz, D. 2001, ApJ, 556, 562

Ciesla, L., Boselli, A., Bendo, G. et al. 2012, A\&A (in preparation)

Dale, D. \& Helou, G. 2002, A\&A A, 576, 159

Draine, B. T. \& Li, A. 2007, ApJ, 657, 810

Elbaz, D. et al. 2011, A\&GA, 533, A119

Kessler, M. F. et al. 1996, A\& $A, 315$, L27

Neugebauer, G. et al. 1984, ApJ, 278, L1

Pilbratt, G. et al. 2010, A\&A A, 518, 3

Werner, M. et al. 2004, ApJS, 154, 1 